\title{
Children with severe asthma can start allergen immunotherapy after controlling asthma with omalizumab: a case series from Poland
}

Iwona Stelmach ${ }^{1}$, Paweł Majak², Joanna Jerzyńska², Magdalena Bojo², Łukasz Cichalewski², Katarzyna Smejda²

${ }^{1}$ Department of Pediatrics and Allergy, N. Copernicus Hospital, Lodz, Poland ${ }^{2}$ Department of Pediatrics and Allergy, Medical University of Lodz, Lodz, Poland

Submitted: 21 March 2014

Accepted: 21 March 2014

Arch Med Sci 2015; 11, 4: 901-904

DOI: 10.5114 /aoms.2015.48546

Copyright (c) 2015 Termedia \& Banach

Anti-lgE treatment reduces exacerbations, symptoms and, in some patients, the dose of inhaled glucocorticoids needed to maintain asthma control [1-3]. Omalizumab, a monoclonal anti-immunoglobulin E antibody, has been successfully used as a supplementary therapy to improve asthma control in children aged $\geq 6$ years with severe persistent allergic asthma who experience frequent symptoms and have asthma exacerbation despite the use of high-dose inhaled corticosteroids and long-acting $\beta_{2}$-agonists (LABA) [4-6]. Omalizumab might also be a treatment option in patients who fail to achieve the intended maintenance dose of specific immunotherapy (SIT) [7, 8]. We conducted an observational study of the clinical experience of maintenance treatment with omalizumab (Xolair, Novartis, Switzerland) in 17 Polish children and adolescents with severe asthma who did not tolerate the allergen immunotherapy prior to the initiation of omalizumab. This was a real-life, open, uncontrolled, observational study. We identified 17 patients aged 7-18 years (not randomly selected) with severe uncontrolled allergic asthma who fulfilled the criteria for anti-lgE therapy and were treated with omalizumab. All patients had multiple allergies; the most clinically important allergens were house dust mite $(n=15)$ and molds $(n=2)$. Five patients had previously qualified for specific immunotherapy (data from medical history), but the maintenance dose could not be reached because of recurrent asthma exacerbations, and SIT was discontinued. Written consent was obtained from all participants and their parents. Omalizumab was administered according to a dosage table that considers the patient' s body weight and total IgE levels determined up to 4 weeks before the first injection of omalizumab. At each visit (every $2-4$ weeks), the physician's overall assessments of treatment, the number of exacerbations and hospitalizations, the use of inhaled corticosteroids controlling asthma and lung function were evaluated. An asthma exacerbation was defined by the presence of at least one of the following: unscheduled patient visit, need for systemic corticosteroids, hospitalization.

The study was approved by the Ethics Committee of the Medical University of Lodz, Poland.

To assess changes in study endpoints, repeated measures analysis of variance was used for parametrically (ANOVA) and non-parametrically (Friedman test) distributed variables. Categorical variables were com-
Corresponding author: Prof. Iwona Stelmach MD, $\mathrm{PhD}$

Department of Pediatrics and Allergy

N. Copernicus Hospital 62 Pabianicka St

93-513 Lodz, Poland Phone: +48 426895972 Fax: +48 426895973 E-mail: alergol@kopernik. lodz.pl 


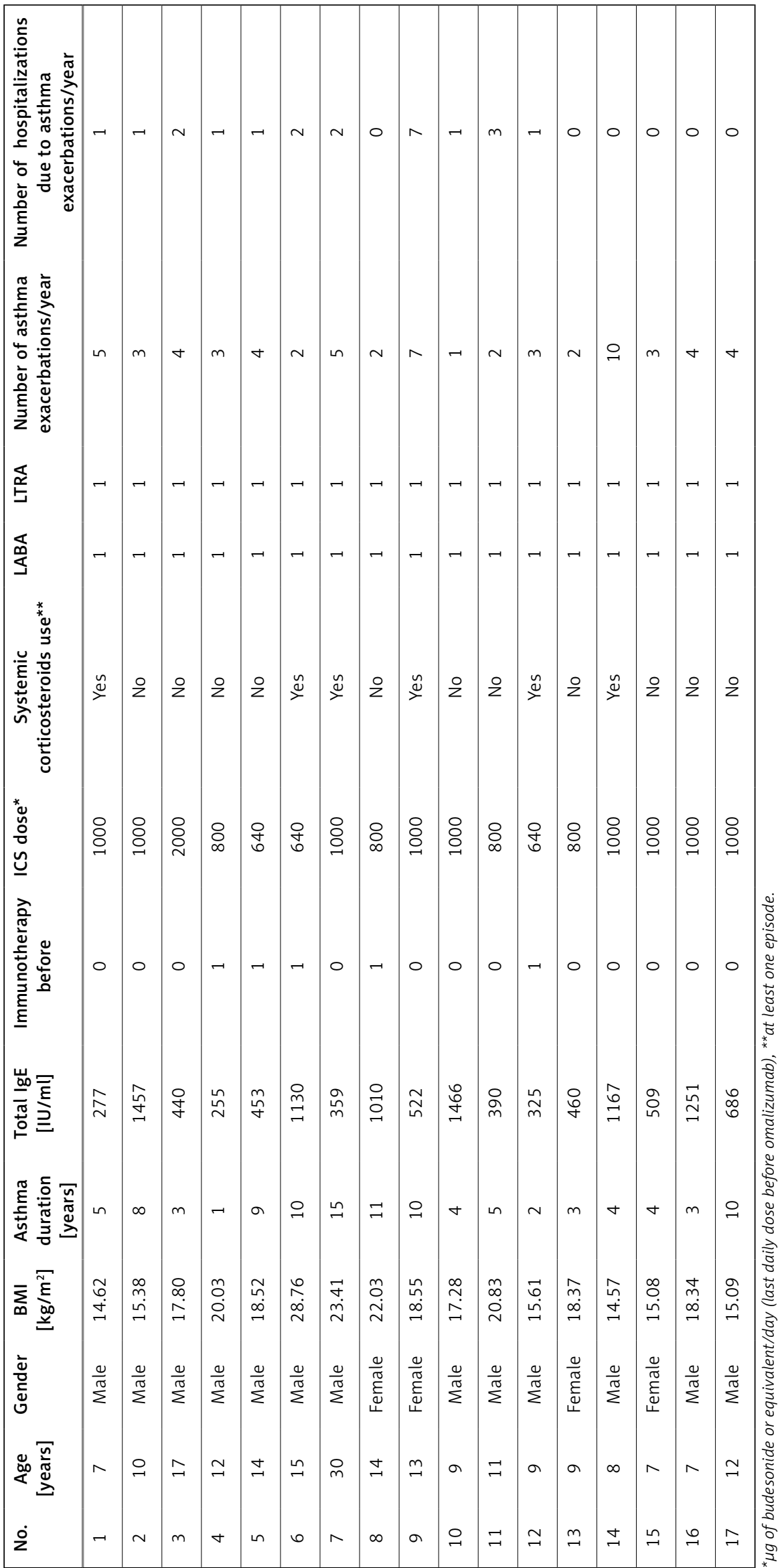


pared with Fisher's exact test. To assess the independent effect of SIT on inhaled corticosteroids (ICS) dose reduction or the number of exacerbations, logistic regression analysis was used. All statistical analyses were performed using StatSoft Statistica for Windows, release 8.0 (StatSoft, Inc., Tulsa, USA). Value of $p<0.05$ was used as a definition of statistical significance.

All 17 patients who received omalizumab for at least 52 weeks were included in the analysis. Patient characteristics are presented in Table I. All patients tolerated omalizumab well. The median number of asthma exacerbations in the 12 months before Xolair initiation was 3 (quartile range: 2-4.5). After 12 months of treatment, the number of asthma exacerbations significantly decreased (Figure 1 A). A similar trend was observed in the number of hospitalizations due to asthma exacerbations (Figure $1 \mathrm{~B}$ ). After 12 months of therapy, the use of ICS had decreased significantly; only 4 patients were treated with the same dose of ICS after 6 months of omalizumab. Relative change from baseline in forced expiratory volume in $1 \mathrm{~s}$ $\left(\right.$ FEV $_{1}$ ) (Figure $1 \mathrm{C}$ ) and daily ICS dose (Figure $1 \mathrm{D}$ ) are shown in figures. After 12 months of therapy, all patients were treated with lower ICS doses; the median ICS dose reduction was $-50 \%$ (quartile range: from $-50 \%$ to $-60 \%)$, and the lowest reduction was $20 \%$. Additionally, we observed a significant decrease in the percentage of patients treated with add-on LABA and/or leukotriene receptor antagonist (LTRA) therapy (Figure $1 \mathrm{E}$ ). In 5 patients, LABA or LTRA therapy was discontinued after 12 months. During the study, SIT was started
A

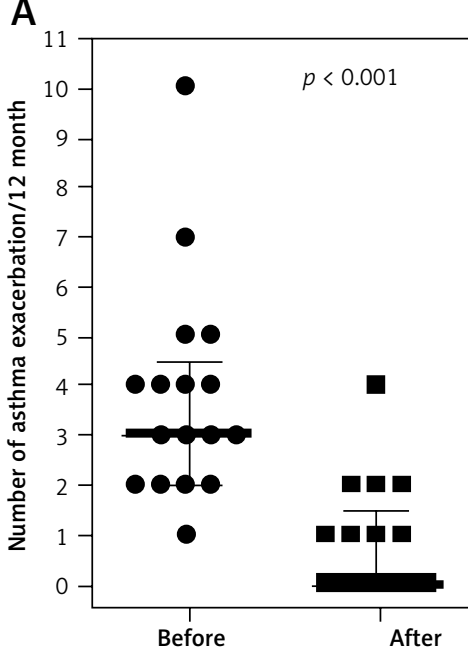

D

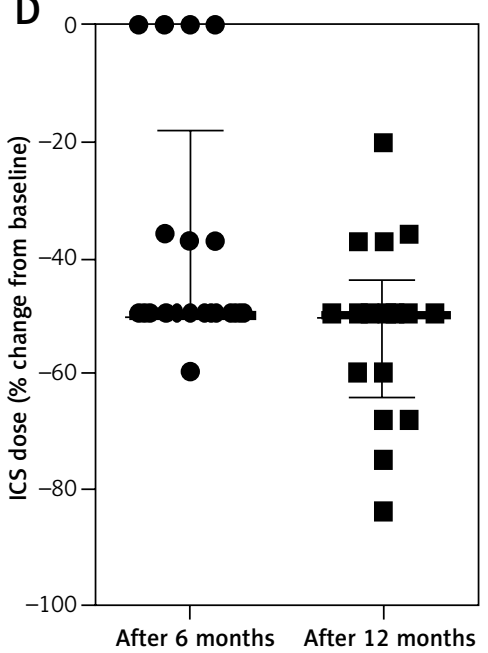

B

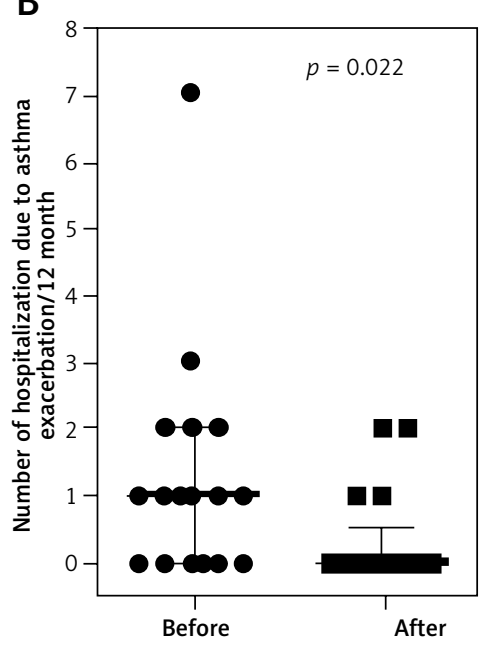

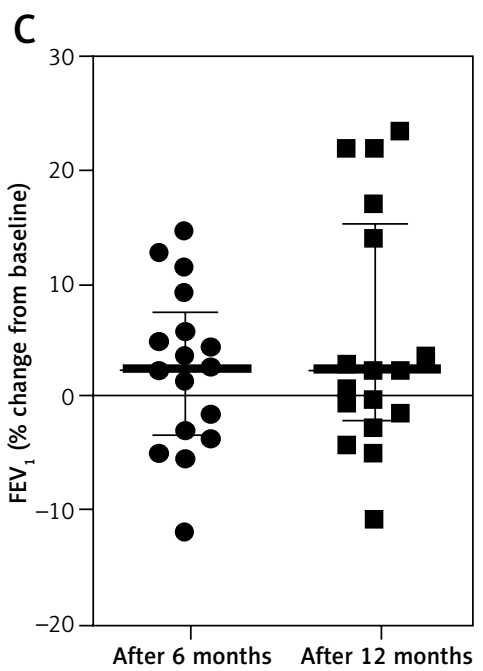

E

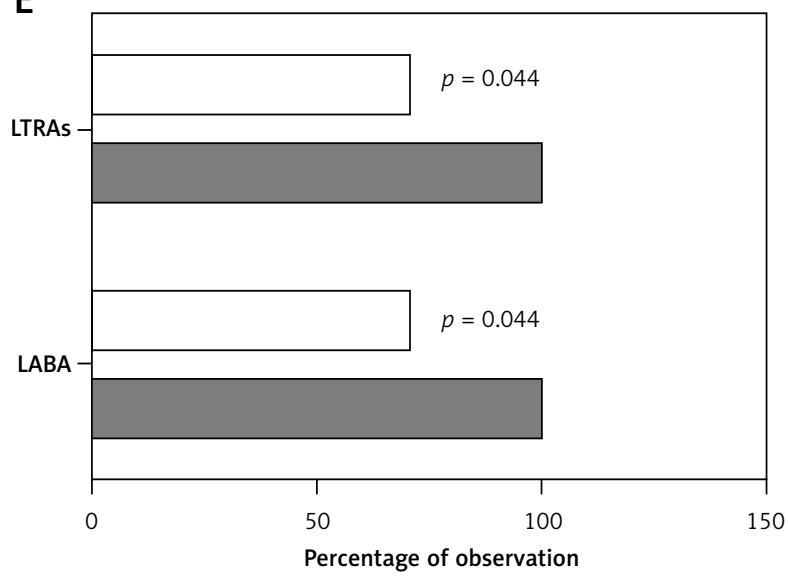

$\square$ Before $\square$ After 12 months

Figure 1. Number of asthma exacerbations (A) and hospitalizations due to asthma exacerbations (B) in each participant 12 months before and 12 months after treatment with omalizumab. Relative change from baseline in FEV $_{1}$ (C) and daily ICS dose (D) after 6 and 12 months in each patient; both parameters were calculated as follows: (value after - value before)/value before $\times 100 \%$. Participants with add-on therapy with montelukast sodium (LTRA) and/or a long-acting- $\beta_{2}$ agonist (LABA) before and after 12 months of omalizumab (E). In Figures A-D data are presented as the value for each participant and with median together with lower and upper quartiles. In Figure $\mathrm{E}$ data are presented as the percentage of observations 
in $12(70.6 \%)$ patients - in 4 (33\%) patients after 3 months, in 5 (42\%) patients after 6 months, and in $3(25 \%)$ patients after 9 months of omalizumab treatment - and was successfully continued according to the SIT scheme. In 5 children who had not tolerated immunotherapy before omalizumab treatment, SIT was introduced after 6 months of treatment and has been successfully continued. Logistic regression analysis did not show any independent effect of SIT on the ICS dose reduction or the number of exacerbations; this subgroup analysis did not have sufficient power.

At present, this study represents non-randomly selected clinical cases treated with omalizumab in Poland. The treatment with omalizumab was clinically effective in all patients: the frequency of exacerbations and the number of hospitalizations were reduced, and there was a significant decrease in steroid use. This effect of omalizumab allowed the initiation of SIT in children with severe asthma. Only 6 months of therapy led to a significant steroid sparing effect by omalizumab. In most of our patients, omalizumab was combined with immunotherapy; we therefore may suspect that the reduction in ICS use will persist after the discontinuation of anti-lgE therapy.

We can compare our results with the landmark study by Kopp et al. [9], in which 16 patients of similar age to those described herein were randomized to receive omalizumab plus placebo or omalizumab plus SIT. The authors reported that omalizumab plus SIT significantly reduced the symptom load in a manner that was clinically relevant when compared with SIT alone. The results of Kopp's and our own study may provide new perspectives for the application of SIT in patients with moderate to severe asthma. The clinical implication of the above findings is that children with moderate to severe allergic asthma who are pre-treated with omalizumab might also benefit in the future from SIT as a causal treatment option. Moreover, the administration of omalizumab prior to SIT reduces the risk of SIT-related systemic reactions and enables more patients to achieve the target SIT maintenance dose [1, 9-12]. Our study group primarily included children younger than 12 years old with relatively short-lasting asthma, which may explain the spectacular modifying effect of omalizumab combined with SIT in most of the included patients.

This real-life study showed that pretreatment with omalizumab should be specifically considered in children with severe allergic asthma to prevent asthma deterioration/exacerbation. This approach allows for the safe and effective initiation of SIT and changes the natural course of severe allergic asthma in children.

\section{Acknowledgments}

The study was self-funded.

\section{Conflict of interest}

The authors declare no conflict of interest.

\section{References}

1. Kopp MV. Role of immunomodulators in allergen-specific immunotherapy. Allergy 2011; 66: 792-7.

2. Busse WW, Morgan WJ, Gergen PJ, et al. Randomized trial of omalizumab (anti-lgE) for asthma in inner-city children. N Engl J Med 2011; 364: 1005-15.

3. Pavord ID, Bush A. Anti-IgE for asthma in inner-city children. N Engl J Med 2011; 364: 2556-7.

4. Brodlie M, McKean MC, Moss S, Spencer DA. The oral corticosteroid-sparing effect of omalizumab in children with severe asthma. Arch Dis Child 2012; 97: 604-9.

5. Mathew J, Aronow WS, Chandy D. Therapeutic options for severe asthma. Arch Med Sci 2012; 8: 589-97.

6. Kupryś-Lipińska I, Kuna P. Loss of asthma control after cessation of omalizumab treatment: real life data. Postep Derm Alergol 2014; 31: 1-5.

7. Kopp MV, Hamelmann E, Zielen S, et al. Combination of omalizumab and specific immunotherapy is superior to immunotherapy in patients with seasonal allergic rhinoconjunctivitis and co-morbid seasonal allergic asthma. Clin Exp Allergy 2009; 39: 271-9.

8. Kopp MV, Hamelman, Bendiks M, et al Transient impact of omalizumab in pollen allergic patients undergoing specific immunotherapy. Pediatr Allergy Immunol 2013; 24: 427-33.

9. Kopp MV, Hamelmann E, Zielenz S, et al.; the DUAL study group. Combination of omalizumab and specific immunotherapy is superior to immunotherapy in patients with seasonal allergic rhinoconjunctivitis and co-morbid seasonal allergic asthma. Clin Exp Allergy 2009; 39: 271-9.

10. Massanari M, Nelson H, Casale T, et al. Effect of pretreatment with omalizumab on the tolerability of specific immunotherapy in allergic asthma. J Allergy Clin Immunol 2010; 125: 383-9.

11. Dąbrowska M, Grabczak EM, Arcimowicz M, et al. Chronic cough - assessment of treatment efficacy based on two questionnaires. Arch Med Sci 2014; 10: 962-9.

12. Marandi Y, Farahi N, Hashjin GS. Asthma: beyond corticosteroid treatment. Arch Med Sci 2014; 9: 521-6. 